\title{
FRATURA-LUXAÇÃO DE LISFRANC: UMA REVISÃO BIBLIOGRÁFICA
}

\section{ARTIGO DE REVISÃO}

NAKAMURA, Gustavo Pazoto ${ }^{1}$

SPAZIANI, Amanda Oliva ${ }^{2}$

BARBOSA, Talita Costa ${ }^{3}$

FERRARI, Beatriz Silva ${ }^{4}$

FERNANDES, Daniel Brito ${ }^{5}$

FRANCISCON, José Antônio ${ }^{6}$

${ }^{1}$ Médico Graduado em Medicina pela Universidade Brasil - Fernandópolis, Brasil. Residente em Ortopedia e Traumatologia no Hospital de Ensino da Santa Casa de Misericórdia de Fernandópolis, Brasil.

${ }^{2}$ Graduanda em Medicina pela Universidade Brasil - Fernandópolis, Brasil.

${ }^{3}$ Graduanda em Medicina pela Universidade Brasil - Fernandópolis, Brasil.

${ }^{4}$ Graduanda em Medicina pela Universidade Brasil - Fernandópolis, Brasil.

${ }^{5}$ Médico Especialista em Ortopedia e Traumatologia pelo Hospital Municipal Dr. Mário Gatti - Campinas, Brasil. Graduado em Medicina pela Fundação Educacional Dom André Arcoverde - Valença, Brasil.

${ }^{6}$ Médico Graduado em Medicina pela Universidade Federal de Uberlândia, Brasil. Residência Médica em Ortopedia e Traumatologia pela Universidade Federal de Uberlândia, Brasil. Preceptor do Curso de Medicina da Universidade Brasil Fernandópolis, Brasil. 
SANTOS, Flávio Henrique Nuevo Benez Dos ${ }^{7}$

NAKAMURA, Gustavo Pazoto. Et al. Fratura-Luxação De Lisfranc: uma revisão bibliográfica. Revista Científica Multidisciplinar Núcleo do Conhecimento. Ano 04, Ed. 09, Vol. 01, pp. 05-17. Setembro de 2019. ISSN: 2448-0959, Link de acesso: https://www.nucleodoconhecimento.com.br/saude/fratura-luxacao

\section{RESUMO}

Objetivo: Esse trabalho visa divulgar um conteúdo científico sobre fratura-luxação de Lisfranc. Metodologia: Para a realização desse trabalho foram consultadas as bases de dado SciELO e LILACS, além da Revista Brasileira de Ortopedia e Traumatologia. Foram selecionados artigos publicados no período compreendido entre 2001 e 2017 escritos em português, inglês ou espanhol. Conclusão: A fratura-luxação de Lisfranc é uma lesão grave, mais frequente em homens e ocasionada por eventos traumáticos de alta e baixa energia. O diagnóstico depende de uma anamnese e exame físico minucioso e para concluir o diagnóstico é necessário solicitar um exame de radiografia. $\mathrm{O}$ tratamento depende do nível de diástase da lesão, podendo optar por tratamento conservador se diástase inferior $2 \mathrm{~mm}$ e cirúrgico se superior que esse valor. As complicações agudas mais preocupantes são as lesões vasculares e a síndrome do compartimento e as crônicas são as deformidades e artrose. A abordagem precoce resulta em um melhor prognóstico.

Palavras-chave:Fraturas ósseas, luxações, Lisfranc.

\footnotetext{
${ }^{7}$ Médico Especialista em Ortopedia e Traumatologia pela Universidade São Francisco - Bragança Paulista, Brasil. Especialista em Medicina Física e Reabilitação pela Rede Lucy Montoro de São Paulo, Brasil. Mestre em Ciências da saúde pela Universidade São Francisco - Bragança Paulista, Brasil.
} 


\section{INTRODUÇÃO}

A lesão de Lisfranc é uma rara fratura-luxação que ocorrem nas articulações tarsometatarsais - conhecida, também, como "articulação de Lisfranc"1. O nome foi dado em homenagem ao médico Jacques Lisfranc de Saint-Martin, francês que realizava amputação dessa articulação durante as guerras de Napoleão, quando soldados caiam do cavalo com o pé preso no estribo. O médico francês alcançou grande reconhecimento como cirurgião, sendo que antes de conhecer a patogênese do espasmo arterial e da isquemia traumática, ele já sabia os perigos da gangrena ${ }^{2}$.

Essa fratura acontece, em muitos casos, em pacientes politraumatizados, correspondendo $0,2 \%$ de todas as fraturas e $9 \%$ dentre as fraturas metacarpais e tarsometatarsais ${ }^{3}$.

A ruptura das articulações tarsometatarsais pode ocorrer direta, com lesões visíveis e consideráveis dos tecidos moles, ou indiretamente, que são mais comuns, que geralmente ocorre devido hiperflexão plantar do pé4. Essa lesão é resultante de um choque de alta energia (acidentes de viação) ou baixa energia (cair de altura), sendo que acidentes motociclísticos, automobilísticos e industriais, somados, correspondem a mais de metade das ocorrências 5 . As consequências da fratura podem ser aguda ou crônica. Dentre as complicações, podem-se destacar síndrome compartimental, lesão nervosa, artrose, pseudo-artrose, necrose da pele, lesão vascular e dor crônica ${ }^{2,3}$.

Inicialmente a fratura de Lisfranc foi relatada por Quénu e Küss ${ }^{6}$ (1909), e posteriormente alterada por Hardcastle ${ }^{7}$ (1982) e, mais recentemente, por Myerson ${ }^{8}$ (1986) ${ }^{1}$. Atualmente dividida em três tópicos: o tipo A tem o movimento das bases de toda estrutura óssea do metatarso em um plano. No tipo $B$ as articulações são luxadas na mesma direção do tipo $\mathrm{A}$, mas nunca envolve todos os raios. $\mathrm{O}$ tipo $\mathrm{C}$, considerado grave, a luxação ocorre em várias direções e pode afetar de forma total os raios, sendo chamada de luxação total. E considerada Tipo $C$ parcial quando tem uma luxação divergente que afeta de um a quatro metatarsos ${ }^{3}$. 
Alguns tipos de tratamentos são possíveis, como abordagem cirúrgica, o uso de órtese e a modificação de calçado ${ }^{9}$. Os fios de Kirschner é uma das formas simples de artrodeses disponíveis; no entanto, mostra grandes índices de falhas ${ }^{10}$. Os parafusos corticais, por sua vez, requerem implantação mais elaborada, mas tem maior taxa de sucesso ${ }^{11}$. Radiografias simples em anteroposterior, oblíquo e de perfil são suficientes para fechar diagnóstico, e se não feitos, pode conduzir a um tratamento inapropriado ${ }^{12}$.

Determinar a percentagem de fraturas de Lisfranc é uma tarefa difícil, pois não são identificadas no setor de urgência, mas em geral gira em torno de $20 \%{ }^{13}$. Essa falha ocorre devido à complexa anatomia do pé, dificuldade na observação da radiografia simples, ou devido abordagem rápida de pacientes politraumatizados ${ }^{14}$. A fratura de Lisfranc não diagnosticada é considerada uma das causas mais comuns de processos por má prática médica contra radiologistas e emergencistas ${ }^{13}$.

\section{METODOLOGIA}

Para a realização desse trabalho foram consultadas as bases de dado SciELO e LILACS, além da Revista Brasileira de Ortopedia e Traumatologia. Foram selecionados artigos publicados no período compreendido entre 2001 e 2017 escritos em português, inglês ou espanhol. Optou-se pelo uso de termos livres, sem necessidade de vocabulário controlado (descritores) e foi utilizada apenas a palavra Lisfranc, ocorrendo assim maior recuperação de referências, garantindo a detecção da maioria dos trabalhos publicados. Foram incluídos artigos acessados de forma gratuita na internet de caráter experimental, observacional ou de revisão literária.

\section{RESULTADOS}

Para melhor elucidação do conhecimento, os tópicos foram divididos entre anatomia, epidemiologia, mecanismo de trauma, classificação, diagnóstico, tratamento, complicações e prognóstico. 


\section{ANATOMIA}

A área é conhecida como articulação Lisfranc representa a transição entre o mediopé e o antepé. A articulação tarsometatársica é composta pela articulação dos três primeiros metatarsos com os ossos cuneiforme medial, intermédio e lateral, além da articulação do quarto e quinto metatarso com o cuboide. A articulação separa a porção dianteira do pé da porção média, sendo referida comumente como articulação de Lisfranc. Atua na supinação e pronação do pé ${ }^{15}$.

O complexo articular da fratura de Lisfranc consiste em cinco articulações tarsometatarsais que ligam o antepé com médio pé. O ligamento Lisfranc é a estrutura que faz a união da base do segundo metatarso ao primeiro cuneiforme, porém existem outros ligamentos que auxiliam nesse complexo. Normalmente o segundo metatarso é o mais atingido. Esse tipo de fratura frequentemente causa instabilidade no médio pé ${ }^{16}$.

O que mantém a área estável é a arquitetura óssea e o suporte ligamentar. Trazendo grande estabilidade ao sistema, estão os cuneiformes e as bases metatarsais, que possui aspecto trapezoidal e o dorso mais alargado. Alguns ligamentos ajudam na estabilidade no plano transverso, por exemplo os ligamentos interósseos plantares e o ligamento de Lisfranc. Existe um suporte dinâmico na região oferecido pelas inserções do tendão fibular e do tendão tibial posterior ${ }^{17}$.

\section{EPIDEMIOLOGIA}

A fratura de Lisfranc (articulação tarsometatársica) é rara, com incidência em torno de 1 para 55000 pessoas por ano 60000 por ano, correspondendo a aproximadamente $0,2 \%$ de todas as fraturas. A maioria das fraturas não são diagnosticadas, o que pode desencadear a dor crônica no pé lesionado, causando osteoartrose e deformidades. Alguns autores consideram que a ausência de diagnóstico em potitraumatizado seja motivo dessa baixa incidência, em torno de $20 \%$ dos casos. Entre vários mecanismos de lesão descrito, o mais frequente é a flexão plantar sobre os metatarsos ${ }^{12,18}$. 
É muito importante para o tratamento, a identificação precoce da fratura juntamente com a redução anatômica, sendo que a fixação estável proporcionam resultados excelentes. A redução anatômica gera resultados satisfatórios em $50 \%$ a $95 \%$ dos pacientes e quando realiza redução não anatômica, o sucesso do tratamento alcança no máximo $30 \%{ }^{12}$.

De acordo com algumas literaturas, há maior ocorrência dessa lesão em homens do que mulheres. Isso ocorre em torno da terceira década de vida e são 2 a 4 vezes mais comuns em homens. Grande maioria das lesões são fechadas $(87,5 \%)$, e que até um terço das lesões são de baixa energia ${ }^{19}$.

O mecanismo de lesão mais prevalente foi acidente automobilístico, com 35,8\% dos casos, seguido por acidentes de motocicleta $(33,3 \%)$, quedas de altura $(23,0 \%)$ e acidentes esportivos (7,77\%), sendo que destes $16,7 \%$ apresentaram traumas diversos. De acordo com a classificação de Quénu e Küss ${ }^{6}, 43 \%$ dos pacientes apresentam lesão do tipo A, $50 \%$ do tipo B e $7 \%$ do tipo C. O tratamento, dependendo do caso, apresentou um tempo médio de internação de 13,8 dias, variando de 0 a 55 dias $^{19}$.

\section{MECANISMO DO TRAUMA}

$\mathrm{Na}$ fratura de Lisfranc, o mecanismo de trauma desenvolve-se por dois tipos, sendo de alta e baixa energia. O primeiro pode resultar de trauma direto e indireto. Uma carga aplicada diretamente no dorso do complexo articular, provocado por esmagamento ou pelo impacto de um objeto no pé, pode resultar em lesões nos ossos ou nos ligamentos através da linha articular, podendo variar o padrão de acordo com a força aplicada. Tal evento pode causar danos aos tecidos moles, comprometendo a sequência do tratamento ${ }^{19}$.

O mecanismo de trauma está associado à força de compressão e, também, aos deslocamentos metatarsofalângicas durante a hiperextensão do pé com as forças axiais, sendo deslocada sentido ipsilateral a forma mais característica de todas as luxações tarsometatarsais. A segunda base do metatarso e $1 \mathrm{~cm}$ proximal a partir do 
primeiro e terceiro metatarso desempenham importante função na estabilização da articulação de Lisfranc ${ }^{20}$.

As lesões são classificadas em diretas quando a lesão é visível nos tecidos moles; ${ }^{4}$ ou indiretas, que são mais frequentes ${ }^{13} \mathrm{e}$ acontecem por acentuada flexão plantar do pé associado a uma força ou carga pelo pé. Muito comum também em movimentos de torção de acentuada energia, num pé em hiperflexão plantar, frequente em pessoas que caem do cavalo e permanecem com o pé preso, ${ }^{21}$ ou quando há um desnível numa curva ${ }^{13}$.

\section{CLASSIFICAÇÃO}

As classificações são utilizadas descrever uma lesão e estabelecer um tratamento adequado $^{22}$.

Uma das classificações mais usadas nas lesões de Lisfranc é a de Quénu e Küss ${ }^{6}$, o qual divide a lesão em 3 tipos, A, B e C. O tipo A envolve a ruptura homolateral, em que todos os metatarsais se movem na mesma direção. A lesão do tipo $B$ há uma ruptura isolada que envolve o primeiro metatarso ou os raios menores. $\mathrm{O}$ tipo $\mathrm{C}$ temse um deslocamento divergente, onde tem-se o deslocamento em direções opostas do primeiro e dos raios menores ${ }^{19}$.

Esta classificação auxilia na comunicação e comparação de dados, mas não é utilizado para definir tratamento ${ }^{14,22}$.

\section{DIAGNÓSTICO}

Para o diagnóstico da lesão de Lisfranc, a anamnese e o exame físico são essenciais. Conhecendo a história clínica e sabendo que uma das queixas frequentes é a dor, a palpação com dor presente entre o primeiro e segundo metatarsais é um achado relevante, mesmo sem fratura com diástase do espaço intermetatarsal ${ }^{23}$. Os achados patognomônicos são dor à palpação, dor a movimentação da articulação tarsometatarsal, equimose plantar na região médio-pé e limitação de sustentação de peso ${ }^{24}$. 
Além disso, pode-se usar dois testes para detectar possíveis fraturas: a compressão lateral e o teste de estabilidade axial entre o primeiro e o segundo metatarso. Quando ambos forem positivos, significa que há a ruptura da articulação de Lisfranc. Um teste específico para essa lesão é a pronação passiva com abdução do antepé com o retropé fixo ${ }^{25}$.

Para concluir o diagnóstico, pode-se fazer uso da radiografia. Se possível, os planos obliquo (O), anteroposterior (AP) e perfil (P) serão feitas com cargas, e a comparação de radiografias entre o pé fraturado e o não fraturado ajudaram a detectar lesões sutis $^{23}$. Na incidência obliqua, a normalidade é o alinhamento entre a face medial do cuboide com a face medial do quarto metatarsal. No anteroposterior, deve haver um alinhamento entre a face medial do cuneiforme intermédio e a face medial do segundo metatarsal. Na posição perfil, é possível identificar a presença de luxações ou subluxações anterior ou posterior das articulações de Lisfranc ${ }^{26,27}$.

Segundo Haapamaki et al, (2004) ${ }^{28}$, a Tomografia Axial Computadorizada (TAC) é um exame de segunda linha para ser feita em pacientes politraumatizados, com lesão de alta energia. Diferentemente da radiografia, ela obtém uma imagem em menos tempo sem a necessidade de um posicionamento específico do pé, tem maior percepção no diagnóstico. Além disso, a TAC detecta fragmentos de ossos e tendões deslocados ${ }^{13}$. Para se obter imagens, a imagem tem que estar orientada no eixo dos metatarsianos quando eles se articulam com o tarso ${ }^{29}$.

Há vários fatores que contribuem para falhas no diagnóstico inicial: A falta de conhecimento da anatomia da articulação tarsometatársica (TMT), radiografias com má qualidade que dificultam sua interpretação, por causa do difícil posicionamento do paciente, e à sobreposição dos ossos nas imagens ajudam na falha do diagnóstico na admissão do paciente ${ }^{30}$. 


\section{TRATAMENTO}

As falhas no diagnóstico correto não permitem o tratamento adequado, levando a ossificação não anatômica e perda de função ${ }^{31}$. O objetivo da terapêutica é a redução anatômica e estabilização da articulação ${ }^{4,22}$.

O tratamento conservador necessita de repouso mínimo 6 semanas com imobilização do pé com gesso, chamada de redução fechada da articulação ${ }^{24}$. A indicação desse tratamento é para lesões com desvio articular menor que $2 \mathrm{~mm}$, confirmado em radiografia específica, ${ }^{24,32,33}$ no entanto, é preciso ter cuidado, pois a redução pode ser perdida após redução do edema das partes moles ${ }^{4,8}$. No seguimento deve ser feito radiografias após 14 dias do começo do tratamento, para confirmar a eficácia da redução e descartar diástase progressiva. A redução deverá ser refeita se forem identificadas alterações e deve ser mantido a imobilização ${ }^{33}$.

As lesões com diástase maiores que $2 \mathrm{~mm}$ entre as bases dos $1^{\circ}$ e $2^{\circ}$ metatarsianos, devem ter terapêutica cirurgica, ${ }^{34}$ para impossibilitar artrose do "midfoot" ou aumentar o desvio articular ${ }^{32}$.

A redução fechada e fixação com fios de Kirchner percutâneos sob controle fluoroscópico, ou redução aberta e fixação interna com parafusos ou fios de Kirchner são as técnicas mais utilizadas ${ }^{4}$.

\section{COMPLICAÇÕES}

$\mathrm{Na}$ fase aguda as principais complicações são a síndrome do compartimento e a lesão vascular, ocorrendo com mais frequência nas lesões por esmagamento. Quando ocorrer a síndrome do compartimento deve ser realizado fasciotomia, com abordagem via medial ou pelas vias dorsais ${ }^{35}$.

Mesmo após a realização de uma terapêutica com redução anatômica, pode correr alterações degenerativas pós-traumáticas, mantendo alterações radiográficas, dor e colapso do arco transversal do pé ${ }^{36}$. As complicações que ocorrem mais tardiamente são as deformidade pós-traumática e a artrose da Lisfranc ${ }^{6,29}$. 


\section{PROGNÓSTICO}

O prognóstico das lesões de Lisfranc é bem controverso, exceto as lesões por esmagamento que vão depender da gravidade da lesão. Mesmo que ocorra as reduções de forma correta, fixação interna e um exame radiográfico pós-operatório de aspecto bom, não é possível correlacionar com um maior sucesso funcional no tratamento $6,8,14,37$.

O fator essencial para uma recuperação funcional é a redução anatômica precoce dessa lesão. As principais complicações são: a permanência de dor, consolidação incorreta, artrose, pé com deformidades, lesões dos tecidos moles e perda da funcionalidade devido ligamentos instáveis ${ }^{13,21,33}$.

\section{CONCLUSÃO}

A fratura-luxação de Lisfranc é uma lesão grave, mais frequente em homens e ocasionada por eventos traumáticos de alta e baixa energia. O diagnóstico depende de uma anamnese e exame físico minucioso e para concluir o diagnóstico é necessário solicitar um exame de radiografia. O tratamento depende do nível de diástase da lesão, podendo optar por tratamento conservador se diástase inferior 2 $\mathrm{mm}$ e cirúrgico se superior que esse valor. As complicações agudas mais preocupantes são as lesões vasculares e a síndrome do compartimento e as crônicas são as deformidades e artrose. A abordagem precoce resulta em um melhor prognóstico.

\section{REFERÊNCIAS}

1. TARCZYńSKA, M. et al. Comparação dos resultados do tratamento de lesões agudas e tardias da articulação de Lisfranc. Acta ortop. bras. [online]. 2013, vol.21, n.6, pp.344-346. ISSN 1413-7852.

2. PEREZ, Q.M.; ALVAREZ, N.R. Luxación tarso-metatarsiana de Lisfranc. A propósito de un caso. Rev. Med. Electrón. [online]. 2011, vol.33, n.1, pp. 155119. ISSN 1684-1824. 
3. HEBERT et al., Ortopedia E Traumatologia: Princípios e Prática. 5. ed. São Paulo: editora Artmed, 2016.

4. STAVLAS, P.; ROBERTS, C.S.; XYPNITOS, F.N.; GIANNOUDIS, P.V. The role of reduction and internal fixation of Lisfranc fracture-dislocations: a systematic review of the literature. Int Orthop. 2010; 34: 1083-91.

5. KORRES, D.S.; PSICHARIS, I.P.; GANDAIFIS, N.; PAPADOPULOS, E.C.; ZOUBOS, A.B.; NIKOLOPOULOS, K. Outcome after anatomic reduction and transfixation with Kirschner wires of Lisfranc joint injuries. Eur J Orthop Surg Traumatol. 2003; 13:85-90.

6. COUGHLIN, M.J.; MANN, R.A.: Surgery of the Foot and Ankle. St. Louis, Mosby Inc., p. 1539, 1999.

7. HARDCASTLE, P.H.; RESCHAUER, R.; KUTSCHA-LISSBERG, E.; SCHOFFMANN, W. Injuries to the tarsometatarsal joint: incidence, classification and treatment. J Bone Joint Surg [Br] 64: 349, 1982.

8. MYERSON, M. The diagnosis and treatment of injuries to the Lisfranc joint complex. Orthop Clin North Am 20: 655, 1989.

9. TENG, AL.; PINZUR, M.S.; LOMASNEY. L.; MAHONEY, L.; HAVEY, R. Functional outcome following anatomic restoration of tarsal-metatarsal fracture dislocation. Foot Ankle Int. 2002; 10:922-6.

10.LEE, C.A.; BIRKEDAL, J.P.; DICKERSON, E.A.; VIETA, P.A.JR.; WEBB, L.X.; TEASDALL, R.D. Stabilization of Lisfranc joint injuries: a biomechanical study. Foot Ankle Int. 2004; 5:365-70.

11. FERNANDES, T.D. et al. Estabilização da artrodese da articulação tarsometatársica: estudo biomecânico. Acta ortop. bras. [online]. 2008, vol.16, n.1, pp.45-48. ISSN 1413-7852.

12.PEREIRA, C.J. et al.Avaliação do tratamento cirúrgico da fratura-luxação de Lisfranc. Acta ortop. bras. [online]. 2008, vol.16, n.2, pp.93-97.

13. GUPTA, R.T.; WADHWA, R.P.; LEARCH, T.J.; HERWICK, S.M. Lisfranc injury: imaging findings for this important but often-missed diagnosis. Curr Probl Diagn Radiol. 2008; 37: 115-26. 
14.MYERSON. M.S.; FISHER, R.T.; BURGESS, A.R.; KENZORA, J.E. Fracture dislocations of the tarsometatarsal joints: end results correlated with pathology and treatment. Foot Ankle. 1986; 6: 225-42.

15.STONE, C. K.; HUMPHRIES, R. L. Current - Medicina de Emergência Diagnóstico e Tratamento -7. Ed.Editora Amgh, 2013.

16. CAMPAGNE, D. Fratura por deslocamento no médio-pé (lesão de Lisfranc), 2014. Disponível em: https://www.msdmanuals.com/ptbr/profissional/les\%C3\%B5es-intoxica\%C3\%A7\%C3\%A3o/fraturas,luxa\%C3\%A7\%C3\%B5es-e-estiramentos/fratura-por-deslocamento-nom\%C3\%A9dio-p\%C3\%A9-les\%C3\%A3o-de-lisfranc. Acesso em: 04 abr. 2019.

17.POZZI, I.; REGINALDO, S.; ALMEIDA, M.V.; FORGAÇA, A. Manual de trauma ortopédico/ SBOT-Sociedade brasileira de ortopedia e traumatologia. São Paulo: Sociedade Brasileira de Ortopedia e Traumatologia, 2011.

18.SILVA, A.P.S.; SHIMBA, L.G.; RIBAS, L.H.B.V.; ALEXANDRE, A.S.; NAVES, V.; DUARTE, JÚNIOR A. Lesão de Turco: diagnóstico e tratamento. Rev. bras. ortop. [Internet]. 2014 ago; 49 (4): 321-327.

19.SOBRADO, M.F.; SAITO, G.H.; SAKAKI, M.H.; PONTIN, P.A.; SANTOS, A.L.G.; FERNANDES, T.D. ESTUDO EPIDEMIOLÓGICO SOBRE LESÕES LISFRANC. Acta ortop. bras. [Internet]. 2017 fev; 25 (1): 44-47.

20. VELÁZQUEZ-VÉLEZ, D.; DURÁN-MARTÍNEZ, N.; PEÑAFORT-GARCÍA, J.A.; ROMERO-PEÑA, A. Controle de Dano do Membro Pélvico em Lesão Complexa no Pé: Relato de Caso. Acta ortop. mex [revista na internet]. 2015 out; 29 (5): 275-279. .

21.KALIA, V.; FISHMAN, E.K.; CARRINO, J.A.; FAYAD, L.M. Epidemiology, imaging, and treatment of Lisfranc fracture-dislocations revisited. Skeletal Radiol. 2012; 41: 129-36.

22.TALARICO, R.H.; HAMILTON, G.A.; FORD, L.A.; RUSH, S.M. Fracture dislocations of the tarsometatarsal joints: Analysis of interrater reliability in using the modified Hardcastle classification system. J Foot Ankle Surg. 2006; 45: 300-3.

23. HARWOOD, M.I.; RAIKIN, S.M. A Lisfranc fracture-dislocation in a football player. J Am Board Fam Pract. 2003;16(1):69-72. 
24. LATTERMANN, C.; GOLDSTEIN, J.L.; WUKICH, D.K.; LEE, S.; BACH, B.R.JR. Practical management of Lisfranc injuries in athletes. Clin J Sport Med. 2007; 17: 311-5.

25. HUNT, S.A.; ROPIAK, C.; TEJWANI, N.C. Lisfranc joint injuries: diagnosis and treatment. Am J Orthop (Belle Mead NJ). 2006;35(8):376-85.

26. PERRON, A.D.; BRADY, W.J.; KEATS, T.E. Orthopedic pitfalls in the ED: Lisfranc fracture-dislocation. Am J Emerg Med. 2001;19(1):71-5.

27.THOMPSON, M.C.; MORMINO, M.A. Injury to the tarsometatarsal joint complex. J Am Acad Orthop Surg. 2003;11(4):260-7.

28. HAAPAMAKI, V.; KIURU, M.; KOSKINEN, S. Lisfranc fracture-dislocation in patients with multiple trauma: diagnosis with multidetector computed tomography. Foot Ankle Int. 2004; 25: 614-9.

29.SANTIN, R. A. L.; FERREIRA, R. C.; MERCADANTE, M. T.; Lesões da articulação de Lisfranc. Rev Bras Ortop, São Paulo, vol. 36, № 4, abril, 2001.

30.VAZ, J.B.P. Fractura de Lisfranc: controvérsias do diagnóstico e tratamento. 2012.

31. RAMMELT, S.; SCHNEIDERS, W.; SCHIKORE, H.; HOLCH, M.; HEINECK, J.; ZWIPP, H. Primary open reduction and fixation compared with delayed corrective arthrodesis in the treatment of tarsometatarsal (Lisfranc) fracture dislocation. J Bone Joint Surg Br. 2008; 90: 1499-506.

32. SCOLARO, J.; AHN, J.; MEHTA, S. Lisfranc fracture dislocations. Clin Orthop Relat Res. 2011; 469: 2078-80.

33. DESMOND, E.A.; CHOU, L.B. Current concepts review: Lisfranc injuries. Foot Ankle Int. 2006; 27: 653-60.

34.SANDS, A.K.; GROSE, A. Lisfranc injuries. Injury. 2004; 35 Suppl 2: SB71-6. 35. MYERSON, M. Diagnosis and treatment of compartment syndrome of the foot. Orthopedics 13: 711, 1990.

36. ARNTZ, C.T.; HANSEN, S.T.JR. Dislocations and fracture dislocations of the tarsometatarsal joints. Orthop Clin North Am. 1987; 18: 105-14

37.TURCO, V.J.; SPINELLA, A.J. Tarsometatarsal dislocation - Lisfranc injury. Foot Ankle 2: 362, 1982. 
Enviado: Maio, 2019.

Aprovado: Setembro, 2019. 